\title{
COMBINING SUPER-RESOLUTION ALGORITHM (GAUSSIAN DENOISING AND KERNEL BLURRING) AND COMPARING WITH CAMERA SUPER- RESOLUTION
}

\author{
Muhamad Ghofur ${ }^{1}$, Tjong Wan Sen ${ }^{2}$ \\ ${ }^{1}$ Information Technology, Faculty of Computing, President University, Bekasi, Indonesia \\ muhamad.ghofur@student.president.ac.id \\ ${ }^{2}$ Information Technology, Faculty of Computing, President University, Bekasi, Indonesia \\ wansen@president.ac.id
}

\begin{abstract}
A good Super Resolution (SR) algorithm is one of the key successes to filter frequency that creates noise to a picture. Previous research that has published was concluded the Camera SR is the best algorithm to filter this frequency based on their Peak Signal to Noise Ratio (PSNR) and Mean Square Error (MSE) results. However, the current approach to achieving high resolution have not yielded enough signal to filter unwanted pixel. Hence, there is a need to find a better approach to those leads to higher resolution through lower noise reduction. To fulfill this need, this thesis proposed to utilize two proven SR algorithms; Gaussian Denoising and Kernel Blurring. This thesis will not only be obtaining these two existing algorithms in a stand-alone form but hence the combination of them (two combinations) will also be obtained as the new possible algorithms that can be utilized to filter frequency that create noise to a picture. To reach the research objective, the method that will be used is by training a total of four algorithms one by one to a public data set that contains 200 pictures and gets the PSNR and MSE results of each algorithm. Comprehensive experimental results show that all those four SR algorithms outperform previous SR algorithms in commonly used data set with variously higher PSNR by $21 \%$ and lower MSE by $5 \%$.
\end{abstract}

Keywords: Camera; Image; Resolution.

\section{INTRODUCTION}

Many jobs require people to use High Resolution (HR) images. However, not all image capture media can get high-resolution images. Many image capture media still produce Low Resolution (LR) images. One of the causes of the resulting image still has a low resolution is due to noise interference that occurs in the resulting image [1]. The noise disturbance occurs due to interference with high-frequency signals when someone takes pictures. A good Super Resolution (SR) algorithm is one of the keys to success in filtering frequencies that can cause noise in the image [2].

There are several ways to run the SR algorithm, from the most frequently researched by researchers, namely by sampling the noise and then doing a network projection on the image. In a study conducted by Adrian Bullat et al regarding SR for facial images, they first used a simple bilinear downsampling method which they called High-toLow Generative Adversarial Network (GAN) to reduce the quality of their facial images. Then that the output from this network is used to train the data to be Low-to High for their SR method by pairing low-resolution images with high-resolution images. The results of this study they were able to get a Peak Signal Noise to Ratio (PSNR) value of $19.30 \mathrm{~dB}$ [3].

In another study that still uses network projections on images conducted by Muhammad Haris et al using the opposite idea by using sampling which is first iterative-up and then down-sampling which they call Deep Back-Projection Network (DBPN). The results of this study they were able to get a PSNR value of $25.50 \mathrm{~dB}$ [4], another study was also carried out by Yulun Zhang et al using the Urban100 data set and a method they called a Residual in Residual (RIR) to form a very deep network. The results of this study they were able to get a PSNR value of $29.04 \mathrm{~dB}$ [5].

Based on the results of the latest research on the 
SR algorithm to filter frequencies that cause noise in the image, it was concluded that Camera SR is the best algorithm currently for filtering these frequencies, when viewed from the PSNR and Mean Square Error (MSE) values obtained. In this study, the average value obtained by Camera SR for PSNR is $30.09 \mathrm{~dB}$ and MSE is 1112.32 [6]. However, if you look at the PSNR results for every single data, it turns out that there is still a single data that gets a PSNR value below $30 \mathrm{~dB}$. While the requirements for an SR algorithm to be able to filter noise optimally if it has a PSNR value above $30 \mathrm{~dB}$.

In addition to several SR algorithms described above, there are 2 algorithms that theoretically and research have proven can be used to filter frequencies that can cause noise in the image. The two proven SR algorithms are Gaussian Denoising [7 - 13] and Kernel Blurring [14 - 20]. The latest research conducted by Ali Awad in 2019 is to use the Gaussian Denoising method, which is divided into 2 stages, namely first for images affected by noise to be smoothed by eliminating noises that have certain information values that affect the information value of their neighbors. Then after that, the remaining noise will be removed gradually. In the process, the two stages are divided into 3 processes, the first process is to divide the image into image patches (image patches), the second process divides the image value into 2 types, namely the type with noise value and the type with information value. The third is that the pixels with noise values will be removed. The results of this study obtained a PSNR value of $33.16 \mathrm{~dB}$ [21]. Meanwhile, Wenming Yang et al in 2017 conducted a study using the Kernel Regression algorithm, namely by linking the low-resolution coding coefficients with high-resolution coding to retrieve and map the non-linear inner relationships between them [22]. Therefore, the two algorithms that already exist and are proven to be able to filter frequencies that cause noise can be optimized for image processing, especially SR in the future.

\section{METHOD}

The following is a research flow process regarding the use of the SR algorithm with several indicators which can be seen in Fig 1 below:

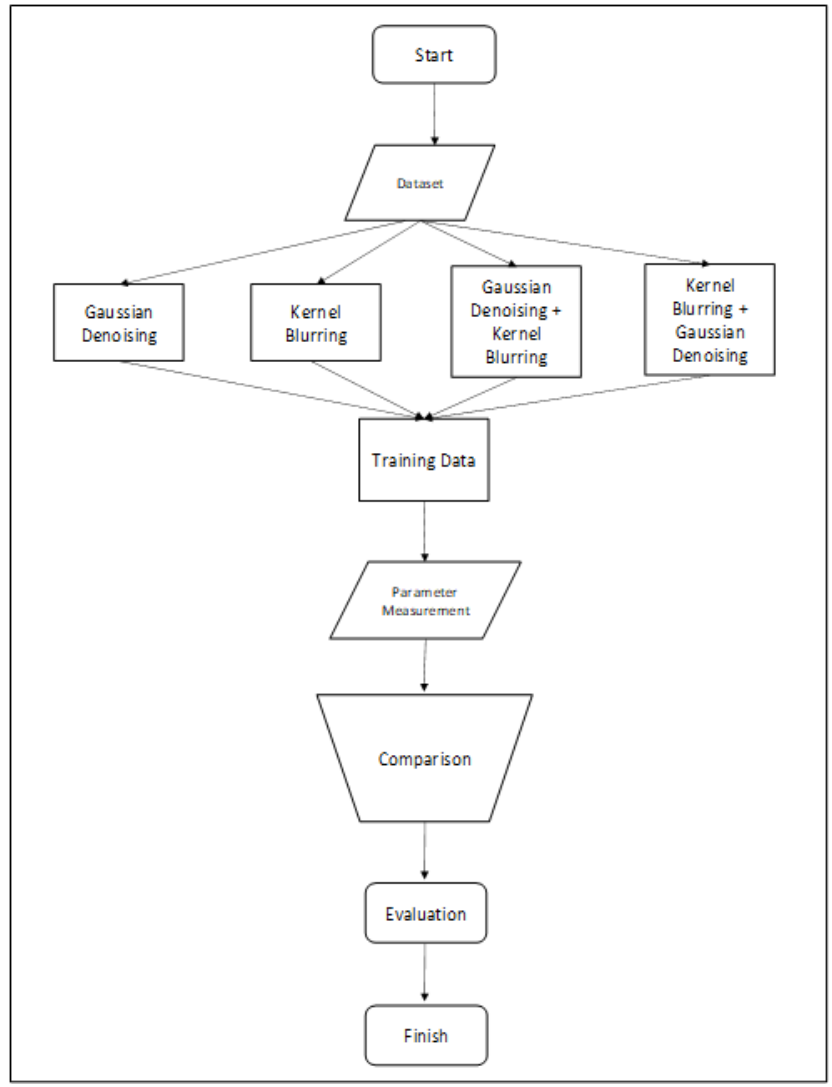

Fig. 1. Research Flow Process

The explanation of Fig 1 regarding the research flow process to be carried out is first is study some pieces of literature that were taken from trusted journal websites such as from IEEE, Science direct, and others, then the literature is reviewed as a basis for finding a research topic. Secondly is data collection is taken from image data before being manipulated. After that, the third is the data is checked to determine whether the data can be used as a research dataset. The data to be taken is public image data before image manipulation is carried out. The fourth one is the training process that will be carried out using the Gaussian Denoising, Kernel Blurring, Gaussian Denoising + Kernel Blurring, and Kernel Blurring + Gaussian Denoising algorithms to obtain Training Data, which is an image of the dataset that has been manipulated by the four algorithms. Finally, the fifth or the last process is checked using Parameter Measurement to find the best PSNR and MSE.

\section{A. Data Collection}

The following is the flow process of data collection methods that will be carried out for matching image data with previous research so that 
the results can later be compared according to the measurement parameters that have been set.

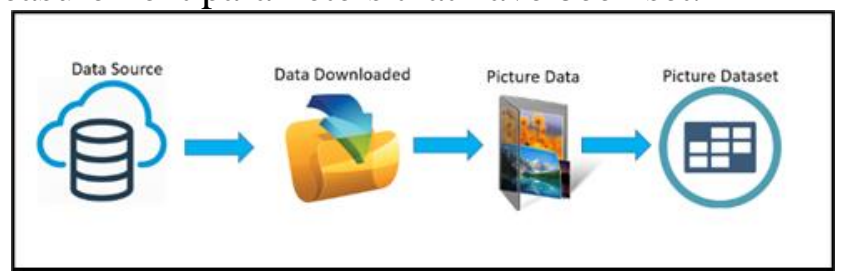

Fig. 2. Data Collection

As in Fig 2 where the image data is taken from the public City100 dataset, with address https://github.com/ngchc/CameraSR/tree/master/Cit $y 100$, then the data is downloaded for a total of 10 GB and after that, it is entered into an excel table with the given 2 labels. The Figure 3 is an example of processed dataset pictures.

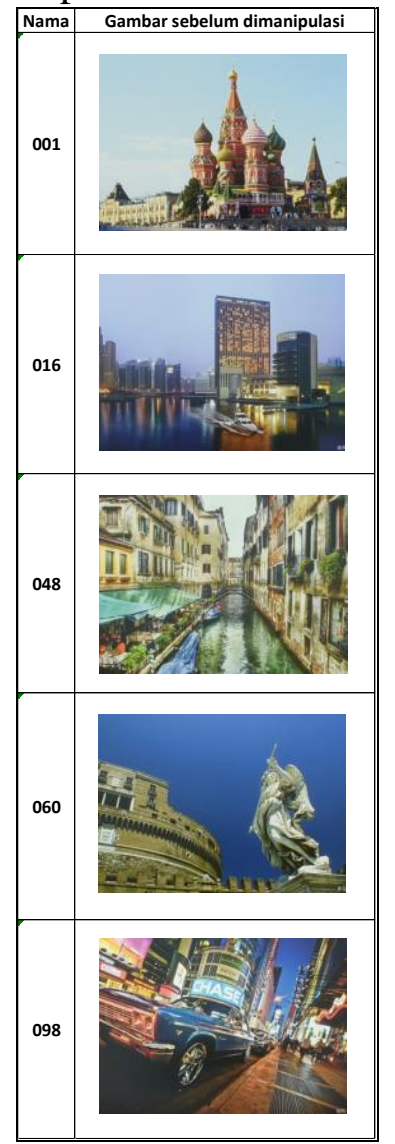

Fig. 3. Picture dataset example

\section{B. Training Model}

The data training model built is an image processing model where later the image dataset will be directly processed using 4 types of SR algorithms, namely Gaussian Denoising, Kernel Blurring, Gaussian Denoising + Kernel Blurring, and Kernel Blurring + Gaussian Denoising.

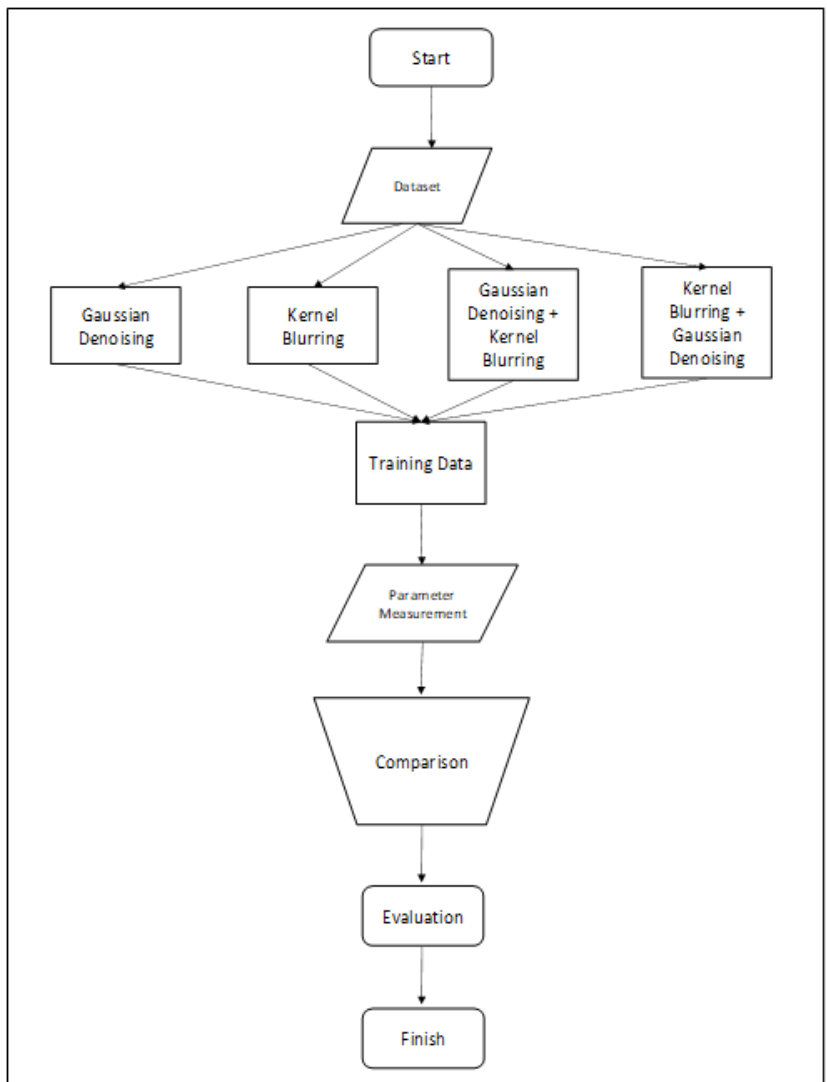

Fig. 4. Training model

\section{Measurement}

At this stage is to take measurements for each experiment carried out to see, compare and decide which SR algorithm is better. Measurements in this study using MSE and PSNR.

Mean Square Error (MSE)

MSE is the mean squared error between the actual value and the forecast value. MSE is also a good parameter to measure the similarity of 2 images. Suppose we have 2 images $h$ and $f$ with dimensions that are $\mathrm{MxN}$, then the way to calculate the MSE is to subtract the value of the image data before being manipulated with the image data after being manipulated and the results are squared (squared) then summed as a whole and divide by the amount of existing data.

$$
\begin{aligned}
M S E & =\frac{\sum_{x=1}^{M} \sum_{y=1}^{N} e^{2}(x, y)}{M \cdot N} \\
& =\frac{\sum_{x=1}^{M} \sum_{y=1}^{N}[h(x, y)-f(x, y)]^{2}}{M . N}
\end{aligned}
$$

Peak Signal To Noise Ratio (PSNR)

PSNR is the ratio of the maximum value of the signal measured by the amount of noise that affects 
the signal. PSNR is measured in $\mathrm{dB}$ units. PSNR is used to compare the image quality before and after the message is inserted (manipulated).

$$
P S N R=10 \log _{10}\left(\frac{C_{\max }^{2}}{M S E}\right)
$$

\section{RESULT AND DISCUSSION}

\section{A. Gaussian Denoising Training}

At this stage, the image data before being manipulated will be trained using the Gaussian Denoising algorithm to form image training data after being manipulated using the Gaussian Denoising algorithm so that the image data will be obtained as shown in Figure 5.

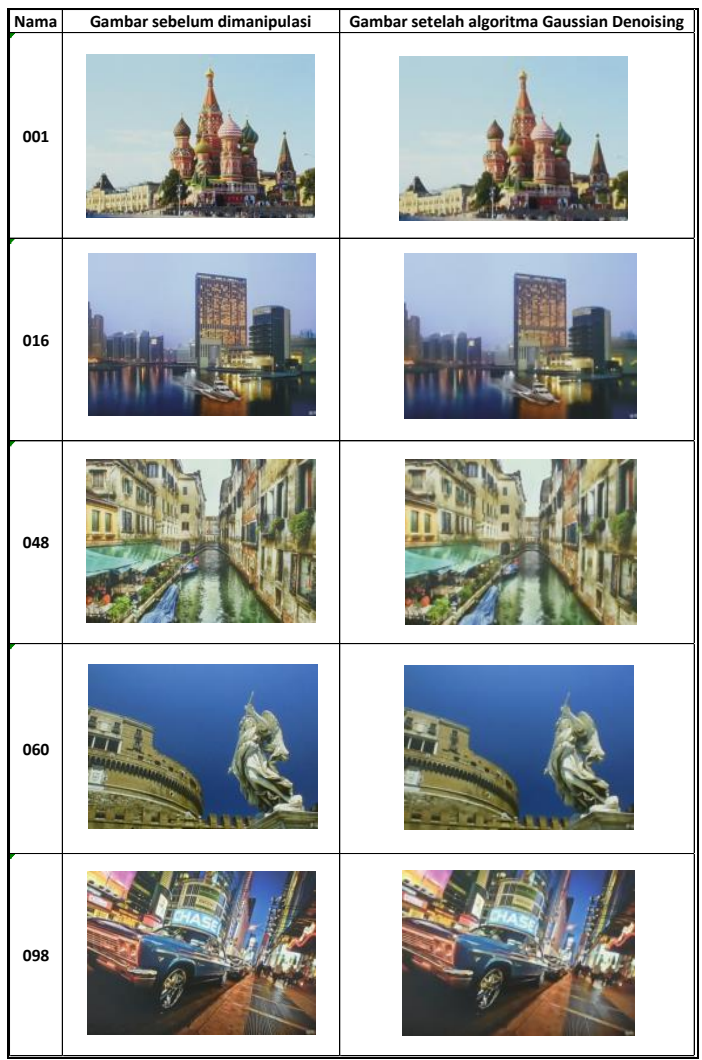

Fig. 5. Picture dataset after denoising

Based on the test results obtained optimal results when the standard deviation $(\sigma)$ of the Gaussian Denoising algorithm is 25.

\section{B. Kernel Blurring Training}

At this stage, the image data before being manipulated will be trained using the Kernel Blurring algorithm to form image training data after being manipulated using the Kernel Blurring algorithm so that image training data will be obtained as shown in Figure 6.

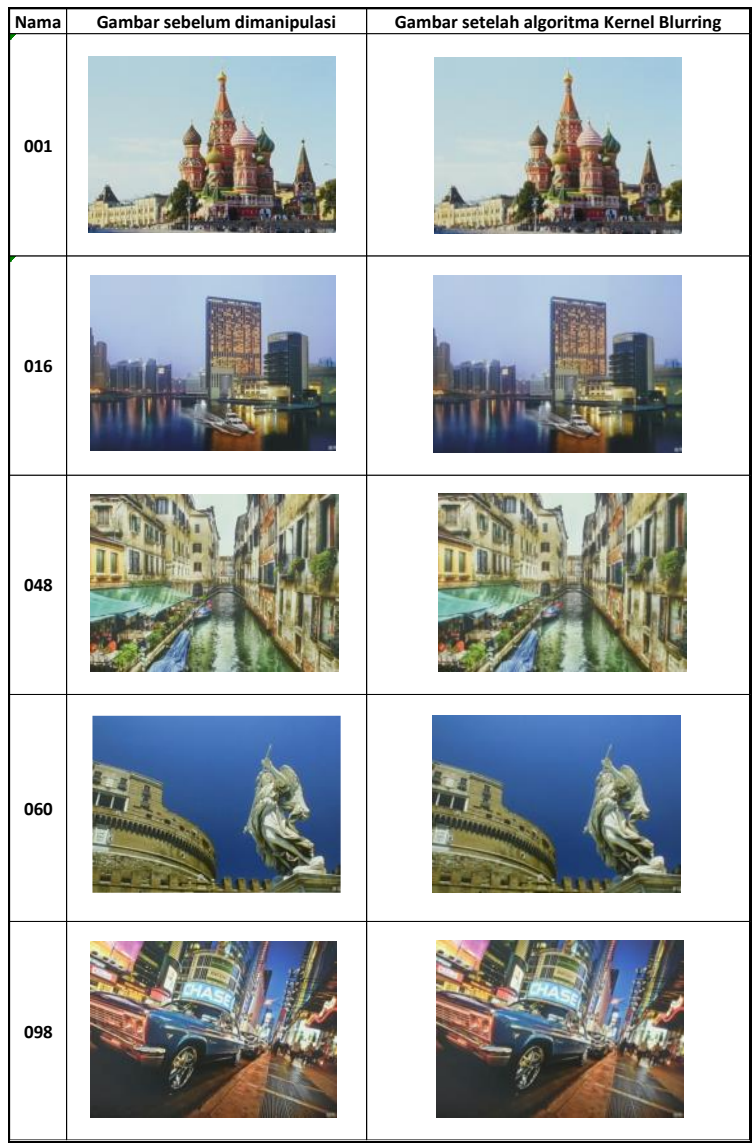

Fig. 6. Picture dataset after blurring

Based on the test results obtained optimal results when the kernel matrix $(h(x, y))$ used in the convolution process of the Kernel Blurring algorithm is $3 \times 3(\mathrm{~h}(3,3))$.

\section{Denoising + Blurring Training}

At this stage, the image data before being manipulated will be trained using the Gaussian Denoising algorithm first so that image training data is formed after being manipulated using the Gaussian Denoising algorithm, after that the manipulated image will be retrained using the Kernel Blurring algorithm so that image training data will be obtained after combined between Gaussian Denoising algorithm with Kernel Blurring as shown in Figure 7.

\section{Blurring + Denoising Training}

At this stage, the image data before being manipulated will be trained using the Kernel Blurring algorithm first so that image training data is formed after being manipulated using the Kernel Blurring algorithm, after that the manipulated image will be retrained using the Gaussian Denoising 
algorithm so that image training data will be obtained after combined between Kernel Blurring algorithm with Gaussian Denoising as shown in Figure 8.

Figure 9 is the results of the MSE test where the lowest MSE value is the result of training data using the Kernel Blurring algorithm.

Figure 10 is the results of the PSNR test where the highest PSNR value is the result of training data using the Kernel Blurring algorithm.

Table 1 is a comparison table of experimental results for several MSE and PSNR measurement parameters.

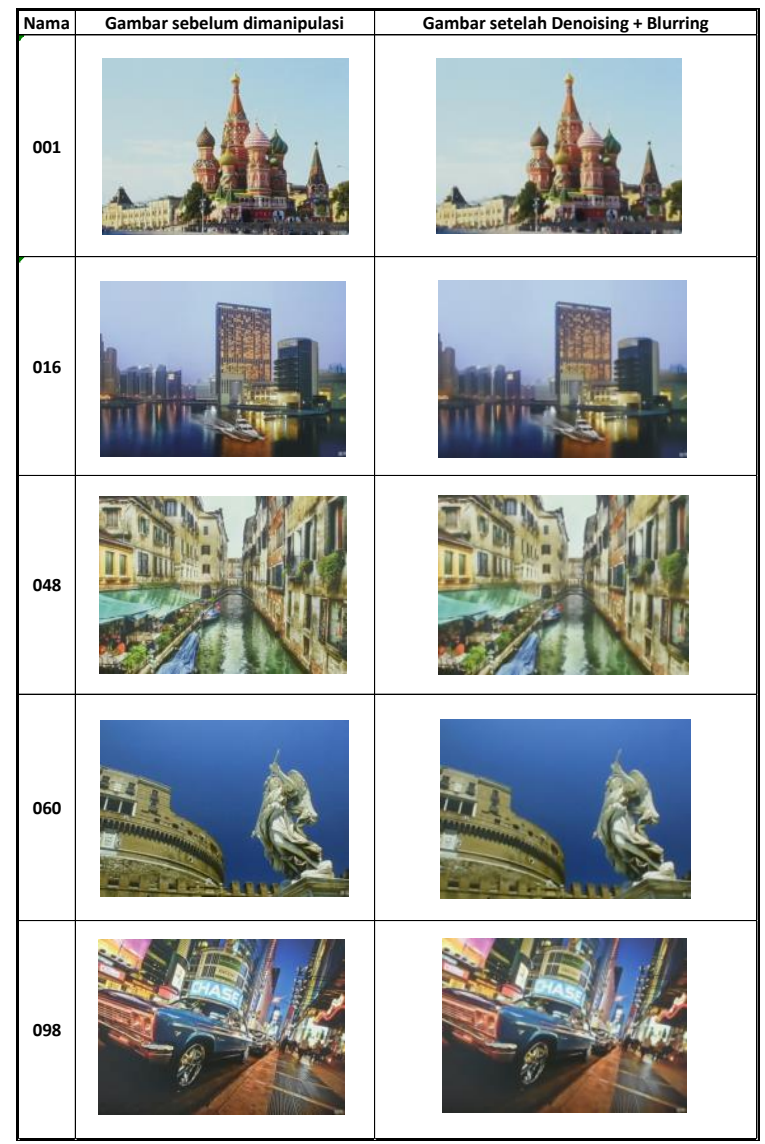

Fig. 7. Picture dataset after denoising + blurring

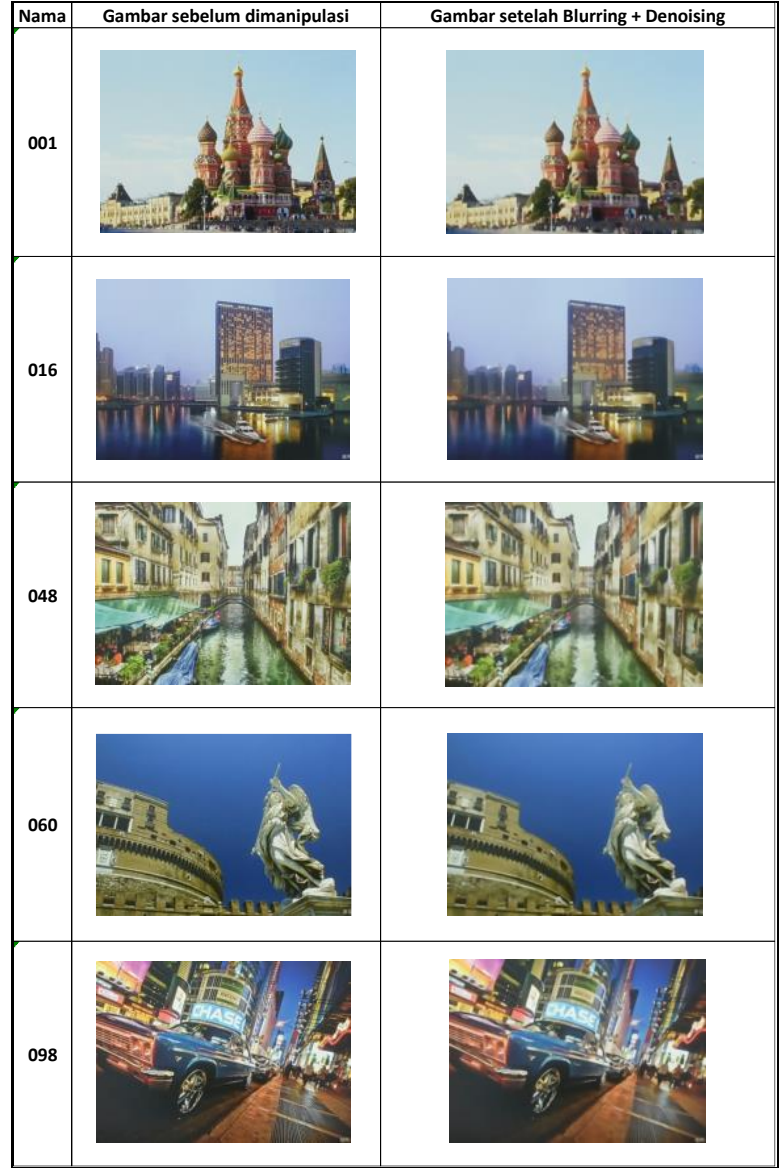

Fig. 8. Picture dataset after blurring + denoising
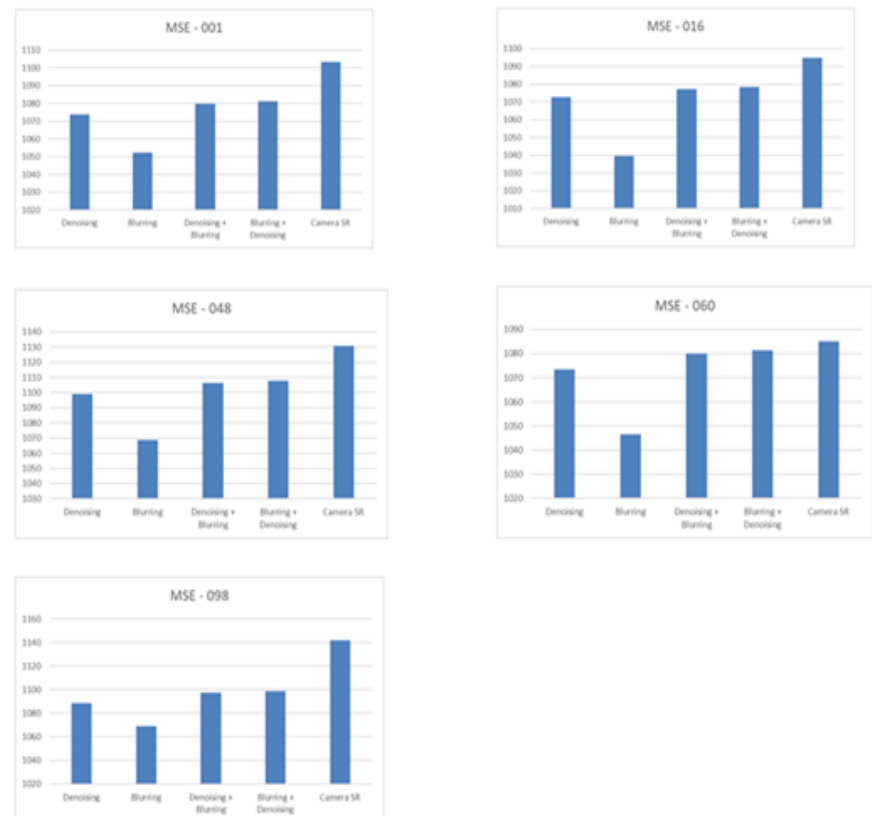

Fig. 9. MSE test result 


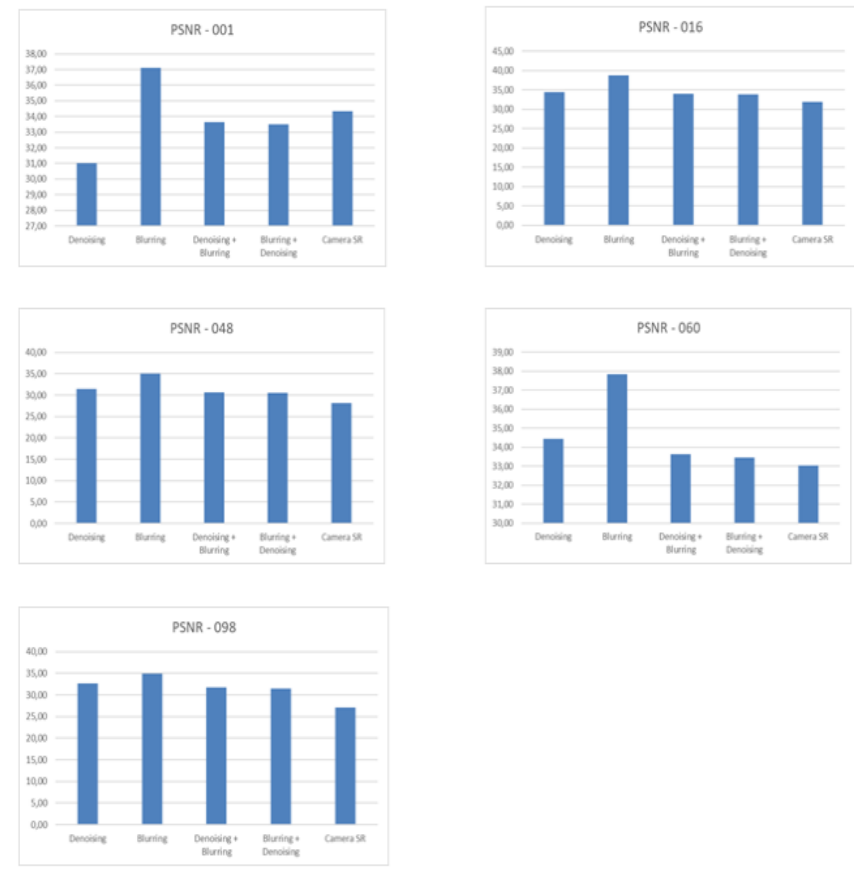

FIG. 10. PSNR TEST RESULT

TABLE I

MSE and PSNR comparison

\begin{tabular}{|l|r|r|}
\hline \multirow{2}{*}{ Algoritma } & \multicolumn{2}{|c|}{ Parameter } \\
\cline { 2 - 3 } Denoising & 1077,24 & 33,98 \\
\hline Blurring & 1058,22 & 36,34 \\
\hline Denoising + Blurring & 1084,57 & 33,12 \\
\hline Blurring + Denoising & 1085,15 & 33,06 \\
\hline Camera SR & 1112,32 & 30,09 \\
\hline
\end{tabular}

\section{CONCLUSION}

The training model in this study uses 2 measurement parameters, namely PSNR and MSE. The results obtained are that from the PSNR and MSE values, the Kernel Blurring algorithm is better than the Camera SR algorithm with a difference of $21 \%$ for PSNR and $-5 \%$ for MSE. The test results for Kernel Blurring obtained PSNR values of $36.34 \mathrm{~dB}$ and MSE of 1,058.22 while Camera SR itself obtained PSNR values of $30.09 \mathrm{~dB}$ and MSE of $1,112.32$. However, when compared visually, the image produced by Kernel Blurring is blurrier than the original image.

\section{ACKNOWLEDGMENT}

I would like to thank Dr. Tjong Wan Sen, my thesis advisor, for his many suggestions and constant support during this research. Mr. Wan Sen is also a computer vision lecturer - a subject that finally I have chosen as my thesis topic.

\section{REFERENCES}

[1] Kai Zhang, Wang meng Zuo, and Lei Zhang. Learning a single convolutional super-resolution network for multiple degradations. In CVPR, 2018.

[2] Xin Deng. Enhancing image quality via style transfer for single image super-resolution. IEEE Signal Processing Letters, 25(4):571-575, 2018.

[3] Adrian Bulat and Georgios Tzimiropoulos. Super-fan: Integrated facial landmark localization and super-resolution of real-world low resolution faces in arbitrary poses with gans. In CVPR, 2018.

[4] Muhammad Haris, Gregory Shakhnarovich, and Norimichi Ukita. Deep back-projection networks for super-resolution. In CVPR, 2018.

[5] Yulun Zhang, Kunpeng Li, Kai Li, Lichen Wang, Bineng Zhong, and Yun Fu. Image super-resolution using very deep residual channel attention networks. In ECCV, 2018.

[6] Chang Chen, Zhiwei Xiong, Xinmei Tian, Zheng-Jun Zha and Feng Wu. Camera Lens Super-Resolution. In CVPR, 2019. [7] M. S. M. Sajjadi, B. Schlkopf, and M. Hirsch. Enhancenet: Single image super-resolution through automated texture synthesis. In ICCV, 2017.

[8] Xintao Wang, Ke Yu, Chao Dong, and Chen Change Loy. Recovering realistic texture in image super-resolution by deep spatial feature transform. In CVPR, 2018.

[9] Jianchao Yang, John Wright and Thomas S. Huang, Yi Ma. Image Super Resolution Via Sparse Representation. In IEEE Transaction on Image Processing, 19(11):2861-2873, November 2010.

[10] Fei Zhou, Wenming Yang, and Qingmin Liao. Interpolation-Based Image Super-resolution Using Multisurface Fitting. IN IEEE Transaction on Image Processing, 21(7):3312-18. July 2012.

[11] Christian Ledig, Lucas Theis, Ferenc Huszr, Jose Caballero, Andrew Cunningham, Alejandro Acosta, Andrew Aitken, Alykhan Tejani, Johannes Totz, Zehan Wang, and Wenzhe Shi. Photo-realistic single image super-resolution using a generative adversarial network. In CVPR, 2017.

[12] Wang X, Wang H, Yang J, Zhang Y. A new method for nonlocal means image denoising using multiple images. In Computational Visual Media, 2(1), 2016.

[13] Mafi M, Izquierdo W, Cabrerizo M, Barreto A, Andrian J, David N and Adjouadi M. Survey on mixed impulse and Gaussian denoising filters. In IET Image Processing, 14(16), 2020.

[14] Jiwon Kim, Jung Kwon Lee, and Kyoung Mu Lee. Accurate image super-resolution using very deep convolutional networks. In CVPR, 2016.

[15] He He and Wan-Chi Siu. Single Image Super-Resolution using Gaussian Process Regression. In Proceedings of the IEEE 
Computer Society Conference on Computer Vision and Pattern Recognition , 5995713:449-456, 2011, Colorado Springs, CO, USA.

[16] Yulun Zhang, Yapeng Tian, Yu Kong, Bineng Zhong, and Yun Fu. Residual dense network for image super-resolution. In CVPR, 2018.

[17] Seyyedyazdi, S and Hassanpour, H. Improving superresolution techniques via employing blurriness information of the image. In International Journal of Engineering, Transactions B: Applications, 31(2), 2018.

[18] Zhao X, Wu Y, Tian J and Zhang H. Single image superresolution via blind blurring estimation and anchored space mapping. In Computational Visual Media, 2(1), 2016.

[19] Angelis G, Gillam J, Kyme A, Fulton R and Meikle S. Image-based modelling of residual blurring in motion corrected small animal PET imaging using motion dependent point spread functions. In Biomedical Physics and Engineering Express, 4(3), 2018.

[20] Jiang X, Wang L, Luo X, Wang S and Luo S. Forwardmotion blurring kernel based on generalized motion blurring model. In Ruan Jian Xue Bao/Journal of Software, 27(8), 2016. [21] Awad A. Denoising images corrupted with impulse, Gaussian, or a mixture of impulse and Gaussian noise. In Engineering Science and Technology, an International Journal, 22(3), 2019.

[22] Yang W, Yuan T, Wang W, Zhou F and Liao Q. SingleImage Super-Resolution by Subdictionary Coding and Kernel Regression. In IEEE Transactions on Systems, Man, and Cybernetics: Systems, 47(9), 2017.

[23] Vandel Maha Putra Salawazo, Desta Putra Jaya Gea, Richard Foarota Gea, Fadhillah Azmi. Implementasi Metode CNN pada Pengenalan Objek Video CCTV. In Jurnal Mantik Penusa, 3(11), 2019.

[24] Mariska Marlia Dwi Purnamawati. Denoising Pada Citra Grayscale Menggunakan Bayesian Tresholding dan Gaussian Noise. In SEMANTIK, 2013.

[25] Tugiono, Hafizah, Asyahri Hadi Nasyuha. Implementasi Pengolahan Citra dengan menggunakan Teknik Konvolusi Untuk Pelembutan Citra (Image Smoothing) dalam Operasi Reduksi Noise. In Jurnal Ilmiah SAINTIKOM, 16(2), 2017.

[26] Wei-Sheng Lai, Jia-Bin Huang, Narendra Ahuja, and MingHsuan Yang. Deep laplacian pyramid networks for fast and accurate super-resolution. In CVPR, 2017.
[27] Bee Lim, Sanghyun Son, Heewon Kim, Seungjun Nah, and Kyoung Mu Lee. Enhanced deep residual networks for single image super-resolution. In CVPR Workshop, 2017.

[28] Assaf Shocher, Nadav Cohen, and Michal Irani. zero-shot super-resolution using deep internal learning. In CVPR, 2018.

[29] Ying Tai, Jian Yang, and Xiaoming Liu. Image super resolution via deep recursive residual network. In CVPR, 2017.

[30] Tong Tong, Gen Li, Xiejie Liu, and Qinquan Gao. Image super-resolution using dense skip connections. In ICCV, 2017.

[31] Z. Xiong, X. Sun, and F. Wu. Robust web image/video super-resolution. IEEE Transactions on Image Processing, 19(8):2017-2028, 2010.

[32] Z. Xiong, D. Xu, X. Sun, and F. Wu. Example-based super resolution with soft information and decision. IEEE Transactions on Multimedia, 15(6):1458-1465, 2013.

[33] Adrian Bulat, Jing Yang, and Georgios Tzimiropoulos. 2018 pirm challenge on perceptual image super-resolution. In ECCV Workshop, 2018.

[34] Justin Johnson, Alexandre Alahi, and Li Fei-Fei. Perceptual losses for real-time style transfer and superresolution. In ECCV, 2016.

[35] Tomer Michaeli and Michal Irani. Nonparametric blind super-resolution. In CVPR, 2013.

[36] Radu Timofte, Shuhang Gu, Jiqing Wu, and Luc Van Gool. Ntire 2018 challenge on single image super-resolution: Methods and results. In CVPR Workshop, 2018.

[37] Adrian Bulat, Jing Yang, and Georgios Tzimiropoulos. To learn image super-resolution, use a gan to learn how to do image degradation first. In ECCV, 2018.

[38] Xiao Zeng and Hua Huang. Super-Resolution Method for Multiview Face Recognition From a Single Image Per Person Using Nonlinear Mappings on Coherent Features. In IEEE Signal Processing Letters, 19(4):195-198, April 2012.

[39] A. Maalouf and M.C. Larabi. Colour image superresolution using geometric grouplets. In IET Image Processing, 6(2):168-180, 2012.

[40] K. Guo X. Yang W. Lin R. and Zhang S. Yu. Learningbased super-resolution method with a combining of both global and local constraints. In IET Image Processing, 6(4):337-34, 2012. 